
ETHICS

Dual-process theories, cognitive decoupling and the outcome-to-intent shift:

A developmental perspective on evolutionary ethics

Gordon P. D. Ingram1 \& Camilo Moreno-Romero

Department of Psychology,

Universidad de los Andes, Colombia

To appear in:

H. De Cruz \& J. De Smedt (Eds.), Empirically engaged evolutionary ethics (New York: Springer).

1 Corresponding author:

Dr Gordon P. D. Ingram

Departamento de Psicología

Universidad de los Andes

Carrera 1 \# 18A-12

Bogotá, Colombia

gp.ingram@uniandes.edu.co 


\title{
Dual-process theories, cognitive decoupling and the outcome-to-intent shift: \\ A developmental perspective on evolutionary ethics
}

\begin{abstract}
Introduction
A central tenet of evolutionary ethics is that as a result of evolutionary processes, humans tend to respond in certain ways to particular moral problems. Various authors (particularly those associated with "dual-process" theories of reasoning) have posited conflicts between "fast", automatic, evolved impulses and more controlled judgements that may be more flexible and respond to culturally determined. In this chapter we argue that the evolutionary sources of automatic moral judgements are quite diverse, and include some intuitive processes (specifically, reading other people's intentions) that are quite sophisticated from a social-cognitive point of view. We further argue that processes of controlled, reflective moral reasoning represent the activity of higher-level process that arbitrate between conflicting inputs from diverse automatic heuristics, in response to normative concerns. The integration and subjugation of automatic responses to more reflective ones is a developmental process that develops at varying rates in different people and in different cultural contexts. To make this argument, we first consider how approaches that represent cognition in terms of two types of processes - including but not limited to the automatic/reflective distinction - can be rendered more sophisticated by a consideration of evolutionary developmental psychology. We then apply this more developmentally aware approach to an extended example of the phenomenon in children's moral development known as the outcome/intent shift. We finish by outlining a model that shows how automatic and controlled processes may be integrated in children's social learning in culturally variable ways.
\end{abstract}

\section{An Evolutionary-Developmental Perspective on Dual-Process Theories of Cognition}

Most evolutionarily inspired approaches to psychology, including moral psychology, view adult cognitive functioning as designed by evolution to solve adult adaptive problems. An example is the moral foundations theory of Haidt and colleagues (Graham et al., 2013), which sees moral cognition as responding to different kinds of triggers, each with relevance for differing aspects of fitness, including care/harm, fairness/cheating, loyalty/betrayal, authority/subversion, and sanctity/degradation (see Suhler \& Churchland, 2011, for an extended critique). All these themes are couched in terms of the decisions that adult 
individuals have to make about how to prioritize the positive part of each dyad and avoid the negative. The same focus on adult cognition, and on selective pressures that impact adults (for example, attracting a mate), is present in evolutionary psychological work on morality by Tooby and Cosmides (2010) and in the "universal moral grammar" proposed by Mikhail (2007) along Chomskyan lines. But such a conception of moral adaptations is a simplification: adult human psychology does not spring to life fully-formed like Athena from the head of Zeus. As Tomasello (2019, p. 22) recently put it:

... the target of natural selection is not adult 'traits,' as in classical accounts, but rather ontogenetic pathways. That is, there is natural selection not just for adult end points but also for the construction process that brings them into existence.

In this chapter, we explore the potential contribution to evolutionary ethics of viewing adult moral cognition as rooted in ontogenetic pathways that develop in children as they mature.

In particular, we suggest that this kind of evolutionary-developmental perspective (as elaborated by Bjorklund \& Pellegrini, 2002; and by Tomasello, 2019) can enrich our understanding of dual-process theories of cognition. Dual-process theories originated from a fusion of several traditions in cognitive science, including the classic study on controlled and automatic attention by Shiffrin and Schneider (1997), the neuroscientific work of Panksepp (2005), and the heuristics-and-biases tradition in behavioral economics developed by Kahneman and Tversky (see Kahneman, 2011, for a popular account). According to dualprocess theorists, the human mind involves two fundamentally different types of cognition. Depending on their origin, these distinctions have received different conceptual labels, such as implicit/explicit, intuitive/deliberative, online/offline, and primary-process/secondaryprocess. Kahneman (2011) tried to unify these distinctions in describing cognition in terms of two "systems": System 1 is "hot", fast, automatic and unconscious, while System 2 is "cold", slow, controlled and conscious. More recently, the older terms "Type 1" and "Type 2" have been preferred, particularly in the work of Evans and Stanovich (2013; see also Evans, 2020; Stanovich, 2011), who argued that Type 1 processes represent a diverse "grab-bag" of automatic processes that are autonomous from one another, and may arise from many different cognitive systems within the mind, rather than forming one coherent system.

Before turning to a consideration of moral development, we address two criticisms that have been levelled quite recently at dual-process theories of cognition in general (earlier influential criticisms, e.g. by Keren \& Schul, 2009, were reviewed by Evans \& Stanovich, 2013). The first criticism is a recurring one that was elaborated most recently by Melnikoff 
and Bargh (2018). They held that the tendency to divide cognitive processes into groups of two is itself an artefact of human binary reasoning styles, without necessarily much basis in reality. They questioned whether there is really much evidence for the claim that cognitive traits such as speed of response, automaticity, impenetrability and evolutionary age cluster together into one coherent system. As stated above, dual-process theorists themselves have gone some way to addressing this with their emphasis on Type 1 processes as a "grab-bag" of automatic responses, an "autonomous set of systems" rather than a single system (Evans \& Stanovich, 2013; see also the reply to Melnikoff \& Bargh by Pennycook, De Neys, Evans, Stanovich, \& Thompson, 2018). These automatic responses encompass both "instinctive" responses to things like loud noises or emotional displays, and learned associations and activities that we once had to consciously attend to but no longer have to do so, such as understanding a written word or riding a bicycle. That is, evolutionarily old processes are necessarily automated, but automated processes are not necessarily old. Another step towards moving away from overly simplistic dichotomies is the suggestion by both Evans and Stanovich that controlled Type 2 processes may be divided into two sub-types: one pertaining to the "algorithmic mind", which follows rules of symbolic processing to arrive at a goal, and the other pertaining to the "reflective mind", which refers to:

the tendency to collect information before making up one's mind, the tendency to seek various points of view before coming to a conclusion, the disposition to think extensively about a problem before responding, the tendency to calibrate the degree of strength of one's opinion to the degree of evidence available, the tendency to think about future consequences before taking action, and the tendency to explicitly weigh pluses and minuses of situations before making a decision.

(Evans \& Stanovich, 2013, p. 230; see also Stanovich, West \& Toplak, 2011). 


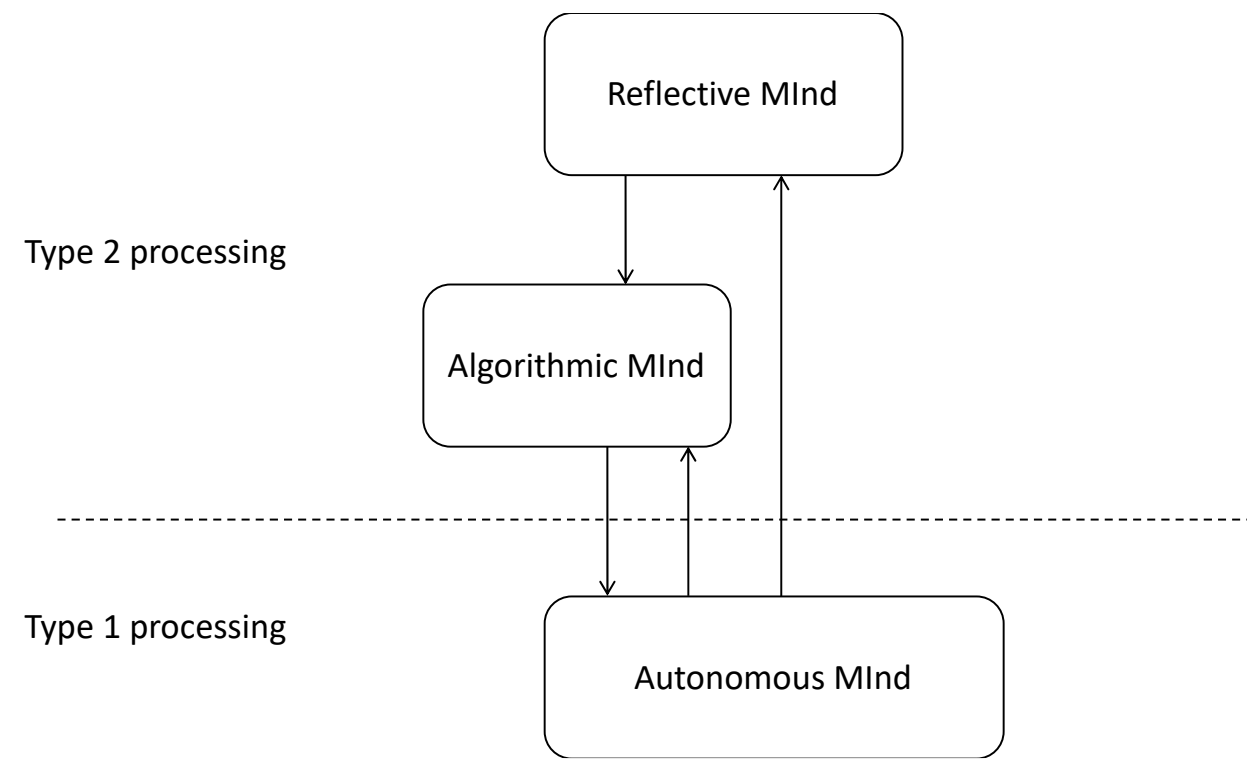

Fig. 1. Stanovich's tripartite model of the mind (after Evans \& Stanovich, 2013, Fig. 1)

A second line of criticism, articulated by Railton (2014), is that intuitive, Type 1 reactions are neither necessarily fast nor innate, because they can embody a lifetime of experience summed up in a single emotional judgement. This process of experience or getting to know one's environment, so that one can have adaptive reactions to it, he calls "attunement". He gave an example of a lawyer who relies on tried and trusted formal rules of setting out her case, until she intuitively realizes that this is not getting through to the jury, and suddenly changes her approach to appeal more to their emotions, with positive results. As with Melnikoff and Bargh's (2018) critique, this points to a rejection of the clustering together of various ways of categorizing cognitive processes, since implicit or intuitive knowledge is not always "fast" or automatic. However, as Pennycook and colleagues (2018, p. 668) pointed out:

DPT [dual-process theory] advocates such as Evans and Stanovich (2013) have explicitly argued against assuming an alignment of the numerous characteristics that have been assigned to so-called 'Type 1' and 'Type 2' processes over the years (see also Evans, 2012; Stanovich et al., 2011). Instead, they distinguish between defining features - those that are used to define the two-types distinction - and typical correlates - those that various researchers have associated with the two-types distinction. 
As pointed out above, the autonomous set of systems captured in the idea of Type 1 processes can include some that are fully automatic and innate, and others that reflect a lifetime of learning via what Railton refers to as "attunement".

Furthermore, an important point not covered by Railton is that the learning that "attunes" intuition often involves deliberative, Type 2 processes: one suffers a setback, reflects on what went wrong, and tries a different approach, which then becomes ingrained if it works better. In his lawyer example, the conscious realization that she was not getting through to her audience led the lawyer to change her behavior in this instance, and probably on certain occasions in the future as well. Railton's example is thus an illustration of how the two types of processing — often presented as in competition with each other, especially in experimental designs - can actually function as a coherent system, ensuring that an individual learns new strategies when it is adaptive to do so. The notion of attunement nevertheless holds promise for looking at how intuitions change during development: can we identify the sorts of social and cognitive processes (including Type 2 processes) responsible for molding or attuning Type 1 intuitive reactions in adaptive ways? One of the goals of this chapter is to show how functional cognitive systems (and the development of these systems through childhood) actually involve elaborate forms of interaction between Type 1 and Type 2 processes, influenced by cultural norms and feedback from the social environment.

Since Type 1 processes are commonly seen as more evolutionarily ancient than Type 2 processes (Stanovich, 2011), it has been natural to assume that they develop earlier in children (Evans, 2011; Barrouillet, 2011). In order to understand them from an evolutionary point of view, and how they interact with later-developing Type 2 processes, we therefore turn to the theoretical perspective of evolutionary developmental psychology. The key insight of evolutionary developmental psychology is that adult human psychology did not evolve as a set of static adaptations to adult life. Instead, what evolved were a set of interconnected developmental processes, having divergent courses, rates and termini in different individuals, with newer - in some sense higher-level and more integrative - cognitive processes layered on top of more situation-specific heuristics.

In introducing an evolutionary-developmental program to psychology, Bjorklund and Pellegrini (2000) argued that a developmental perspective is vital for a better understanding of the evolved psychology of a slow-developing species such as humans. Their approach was inspired by the growing influence of "evo-devo" theory on evolutionary biology, where the idea that developmental biases place important constraints on the evolution of complexity and diversity in all organisms has become widely accepted (Brakefield, 2006; Carroll, 2005; 
Lickliter \& Honeycutt, 2003). An important insight of evolutionary developmental psychology is that certain forms of cognitive functioning can offer adaptive value that is unique to individuals in certain age ranges: these are known as ontogenetic adaptations. In contrast, deferred adaptations are not of much adaptive value to a child at their current point in development, but rather serve as preparations for behavior that will have adaptive value later in the lifespan. According to the principles of evolutionary developmental psychology, then, it could be the case that evolved Type 1 processes are either ontogenetic adaptations to the environments in which children tend to find themselves, or deferred adaptations that serve as building blocks for later-developing algorithmic processes. We return to this idea in the Conclusion.

More recently, Bjorklund (2015) has augmented the concepts of ontogenetic and deferred adaptations with a new concept, that of "evolved probabilistic cognitive mechanisms." This concept is based on the insight that evolution does not tend to specify patterns of behavior very precisely in the genotype of generalist species, like humans, that live in widely varying environments and thus need to show a lot of flexibility in their responses to different stimuli. Instead, adaptations direct an individual's attention to fitnessrelevant stimuli in the environment. Two examples cited by Bjorklund are learning a fear of snakes and spiders (LoBue \& Rakison, 2013), and picking up relevant emotional information from human facial expressions (Farroni, Massaccesi, Menon, \& Johnson, 2007). With their attention directed to the stimuli most relevant to their survival and prosperity (in fitness terms), human children then have an opportunity to use probabilistic social learning to acquire effective responses from other, more knowledgeable individuals who are attending to the same stimuli at that moment in time.

According to this view, then, what evolves in children is a set of interconnected developmental or learning processes, which lead to a gradual layering of acquired cognitive processes on top of innate heuristics (cf. Rochat, 2015). Because learning is probabilistic and the initial amount of attention paid to stimuli varies genetically, these developmental processes have divergent courses, rates and termini in different individuals. This conceptualization recalls Railton's (2014) argument that "intuitive" moral judgements are not crude heuristics, but complex learned responses of the affective system. Although his idea of "attunement" (discussed above), is not meant to capture the development of an evolved cognitive mechanism, it may not be so different from Bjorklund's conception of probabilistic learning. We have instinctive attentional biases for paying attention to faces and other implicit emotional information (Farroni et al., 2007), and then through experience we learn 
probabilistically things like how to read the signs of when people are sympathetic to our argument, when we are provoking hostility, when we are boring them, etc. This intuition becomes a resource that people can then use more reflectively, as in his lawyer example. To give a more systematic example of how evolved probabilistic mechanisms may be similar to processes of attunement, in the rest of the chapter we consider the case of the outcome-tointent shift in moral development.

\section{The Outcome-to-Intent Shift}

Although they have not always phrased cognitive development in terms of two kinds of processes, many developmental psychologists from Piaget onwards have tried to explain how children transition from more automatic (informal, implicit) to more controlled (formal, explicit) types of reasoning. An important theoretical advance was made by Karmiloff-Smith (1992), who explored several examples from non-social cognition of interactions between the two types of processes. She used the idea of "representational redescription" to describe the sort of U-shaped curve that occurs in the development of certain cognitive abilities, where initial implicit performance can actually be better than subsequent explicit performance, until the latter has been calibrated and integrated with implicit skills to yield explicit behavioral mastery (Baylor, 2001; Siegler, 2004). For example, children's use of irregular past tense in common English verbs is marked by an initial predominance of correct performance, apparently due to exact imitation; later, with more linguistic experience, correct performance often decreases due to "overgeneralization" of regular forms (e.g. *maked for made); and finally, the calibration of different sets of rules enables children to use both regular and irregular past tenses correctly (Pauls, Macha, \& Petermann, 2013; Siegler, 2004) .More recently, in the domain of social cognition, a similar pattern has been proposed as an explanation of the puzzling incongruity between apparent implicit expectations of false beliefs from late infancy and the tendency of younger three-year-olds to fail explicit falsebelief tasks (Grosse Wiesmann, Friederichi, Singer, \& Steinbeis, 2017). But the most relevant example for evolutionary ethics - and one for which a U-shaped curve has also recently been proposed (Margoni \& Surian, 2016) — is a feature of children's moral development known as the outcome-to-intent shift.1

1 The term "outcome-to-intent shift" seems to have been coined by Cushman, Sheketoff, Wharton, and Carey (2013). The term used by Piaget (1932) for the prioritizing of outcomes by 
The basic idea of the outcome-to-intent shift is that young children tend to give more weight to the outcome than the intention of an action when making moral judgements, a pattern that is reversed in older children and adults. Historically, the classic demonstration of the phenomenon was by Piaget (1932). He showed that when judging how bad a rule violation is, younger children (before age 8 or 9) tend to focus on what might be called the physical extent of the rule violation's consequences, whereas older children (9-10 years onwards) tend to focus on the intentions behind it. This was in keeping with Piaget's wider perspective on the development of moral reasoning, which he characterized in terms of a shift from heteronomous reasoning (rules are seen as having to be obeyed in all circumstances, with any deviations being bad) to autonomous reasoning (rules are seen as social constructions that are flexible depending on the context, and deviations can be excused in various ways). It is a common misrepresentation of Piaget's work to view these stages as irreversible "phases" through which children pass without any possibility of return. In fact, as with other systems of stages in Piaget's thought, they are more like "styles of reasoning" which are more or less accessible at different ages. Even adults may use heteronomous reasoning quite frequently, for example if they are tired or stressed, or if the social context is one in which they are treated like children. The developmental difference is that adults and older children also have the autonomous style of reasoning available to them as an option, whereas younger children do not. Thus, there may be parallels with the idea of dual processes in adult reasoning.

Experimentally, one tactic that Piaget used to demonstrate the shift between heteronomous and autonomous moral reasoning was to compare good intentions with neutral or negative ones; for example:

There was once a little girl who was called Marie. She wanted to give her mother a nice surprise and cut out a piece of sewing for her. But she didn't know how to use the scissors properly and cut a big hole in her dress.

A little girl called Margaret went and took her mother's scissors one day when her mother was out. She played with them for a bit. Then, as she didn't know how to use them properly, she made a little hole in her dress.

(Piaget, 1932, p. 122)

younger children was "moral realism," but this has fallen into disuse, perhaps due to the risk of confusion with the philosophical position of the same name, which has quite a different meaning. 
Asked "who is naughtier", younger children tended to say the first child, Marie, while older children indicated the second child, Margaret. At this point, it is worth noting that there was an important confound in the example of the two vignettes given above, as in many other examples used by Piaget. In order to judge that Margaret's action is worse, a child had to ignore (inhibit) the greater damage, or harm, done by Marie. In subsequent research it has been more common to control for this factor by comparing negative with neutral or positive intentions while keeping the level of damage in the outcomes constant - that is, comparing failed attempts at rule transgressions versus actual, accidental transgressions-but the underlying principle is the same.

As with much of Piaget's stage-based theory, more careful experimental work from the 1970s onwards (Berg-Cross, 1975; Hebble, 1971; King, 1971; Shultz, Wright, \& Scheifler, 1986) showed that children are capable of using a more advanced stage of reasoning at younger ages than Piaget claimed, while still generally supporting his overall picture of an age-based transition in preferences for the two reasoning styles. It turned out that if the level of damage in the outcome was kept constant, some children as young as 4 years of age would say that the girl with the negative intention was naughtier, or that the girl with the positive intention was less naughty. For example, Zelazo, Helwig, and Lau (1996) found that 3-year-olds based their judgments of acceptability and punishment solely on an action's outcomes, but from 4 years of age children were able to use rules in which both intentions and outcomes were included, particularly if the intentions were negative (see also Helwig, Zelazo, \& Wilson, 2001). Hence the term “outcome-to-intent shift” may be a misnomer. While there does appear to be a shift in emphasis from favoring outcomes to favoring intentions as triggers of moral judgements when the two are pitted against each other, this is often glossed as something like, young children do not assign any importance to intentions when making moral judgements, which is not at all correct. The developmental change that takes place instead seems quite specific to accidents. When confronted with an accidental action that results in more harm/damage but without harmful intent, children do tend to judge it as worse than a failed attempt at harm or damage. As they get older, they come to assign even more weight to intentions even in the face of more damaging outcomes.

\section{Using Dual-Process Models to Explain the Outcome-to-Intent Shift}

This more recent evidence would seem to invalidate Piaget's preferred explanation for the outcome-to-intent shift - that intention-related concepts are simply not available to young children in their moral reasoning, due to a lack of perspective-taking. It seems more that 
information about both intentions and outcomes is always available, but that when the two types of information are in conflict (as with accidents) children initially prioritize the outcome and later prioritize the intention. One way of modeling this is to consider the two types of information as handled by different processes that develop separately. This was the approach of Cushman, Sheketoff, Wharton, and Carey (2013; see also Cushman, 2013), who argued that moral judgement in humans is governed by two processes: a causality-sensitive process that infers whether an agent is physically responsible for an outcome, and an intentionality-sensitive process that infers culpability by considering the intentions behind the agent's actions. They measured both moral judgements of character ("Is $X$ a bad, naughty boy/girl?") and recommendations of punishment (“Should $X$ be punished?") through moral vignettes of a child character either acting with neutral intentions but getting a bad outcome, or acting with negative intentions but failing to achieve these. They hypothesized that the effect of the outcome-to-intent shift would be seen first in abstract judgements of badness, before these intent-based judgements came to constrain punishment decisions. They demonstrated that for accidental wrongs, the outcome-to-intent shift took place in their sample at about the age of 5 for abstract judgements (an earlier age than Piaget had supposed) and about age 7 for punishment recommendations. They also replicated the more recent finding that children tend to judge failed negative intentions harshly from as early childhood (the youngest children in their sample were 4 years old).

This latter, unpredicted, replication was however a problem for Cushman and colleagues' theory, since it showed intent-based moral judgement at an earlier age than they predicted it would appear. That is, there was an asymmetry between children's early negative judgements of failed negative attempts (both abstract judgements and recommendations of punishment) and their later-developing exoneration of accidental wrongs (developing later for punishment than for abstract judgements). They tried to resolve this by suggesting that perhaps children had an automatic negative response to "bad acts," which included the attempt to perform such acts (e.g., an attempt to kick another child):

We suggest that children have an early developing automatic negative reaction to "bad acts", along the same lines as their capacity for their negative reaction to "bad outcomes" that gets harnessed during the preschool years to concepts of naughtiness, punishability, and wrongness (concepts that are not initially differentiated).

(Cushman et al., 2013, p. 18)

However, this could be seen as begging the question, since they provided no theoretical reason why a child would automatically interpret an unsuccessful bad intention as if it were a 
bad act, but not interpret an unsuccessful good intention as if it were a good act. Furthermore, they did not explain why the "constraint" of the intentional process over the causal process developed for abstract judgements sooner than for punishment when children were evaluating accidental wrongs, but was present early on for both abstract judgements and punishment when they interpreted failed attempts at wrongs. If information about both causality and intention is used from as early as 3 years when judging failed negative intentions, it is difficult to explain, using the framework of separate processes, why it is not used until 5 years when judging accidental violations in an abstract way, and not until 7 years when judging whether they deserve punishment.

Our previous consideration of dual-process theories may help us construct a more adequate theoretical explanation for the outcome-to-intent shift, particularly with respect to the change in attitudes for the accidental condition (which as argued above, seems to be the key change during development). Cushman and colleagues referred to their theoretical model as a "two-process" and "dual-system" model, in what seems to be quite a loose usage of the term (following Greene, whom they cited in the article), simply identifying two different processes that respond to different aspects of the same situation, rather than tapping in to the more developed dual-process tradition of theorists like Kahneman, Stanovich, and Evans. Indeed, they explicitly claimed to be agnostic about whether the two types of moral judgement (causal and intentional) that they proposed were implicit or explicit: "the two process model suggests that they operate in parallel among adults and is agnostic about their status as explicit conceptual systems versus automatic processes of moral judgment" (Cushman et al., 2013, p. 9). Use of the term "dual-system" seems just to imply that they had identified two processes that worked independently and responded to different facets of experience. In contrast, here we propose abandoning agnosticism and examining the implications of supposing that these two processes of causal and intentional inference are in fact both automatic.

Research on causal reasoning has revealed an early sensitivity to causal inferences associated with the behavior of both objects and agents (Perner \& Roessler, 2012). Many studies have used a habituation/dishabituation paradigm, in which infants are habituated to a specific type of causal event, and then presented with a different type of event during test (dishabituation) trials. A longer looking time in the dishabituation trials, compared to control trials with another causal form, is taken as evidence of causal inference (Bélanger \& Desroches, 2001). This method has shown that even 4-month-old infants are sensitive to typical spatial and temporal cues about causal motion, which cause dishabituation if they are 
absent (Cohen \& Amsel, 1998; Muentener \& Bonawitz, 2017). Causal inferences in infancy are not restricted to objects: by 6 months of age, infants dishabituated when a person reached for a new object if the same person had reached for a different object in previous trials (Cicchino \& Rakison, 2008). This shows that infants can encode goals of intentional human action with reference to objects, and that automatic causal reasoning may emerge from early statistical learning about interactions between agents and objects (Kushnir, Xu, \& Wellman, 2010; Wu, Gopnik, Richardson, \& Kirkham, 2011).

As these latter studies indicate, we also know that processing of intentions (goals) can happen very early in life, and probably automatically (Woodward, 2009). Even rapid belief ascription via theory of mind may sometimes be automatic, in both infants (Scott \& Baillargeon, 2017) and adults (Schneider, Slaughter, \& Dux, 2017), although the infant results are controversial (Kulke, Reiß, Krist, \& Rakoczy, 2018, described several failures to replicate them). More directly relevant to the outcome-to-intent shift are the moral developmental studies of Hamlin and colleagues (e.g., Hamlin, 2013), which showed that preverbal infants preferred toys/characters with good intentions. For example, 8-month-old infants preferred to play with toys that had accidentally hindered another toy's progress up a slope, or that had tried but failed to help the other toy, rather than with those that had tried to hinder the toy but failed. In other words, the outcome for all the characters in these situations was the same - they failed to reach their goal - but children chose which character to play with based on their supposed intentions (for similar results with preschoolers, see Van de Vondervoort \& Hamlin, 2018). Since 8-month-old infants are generally considered too young to have explicit reasoning about intentional concepts, the assumption is that they were automatically encoding the characters' intentions and using these to make moral judgements.

This kind of automatic, "sympathetic" reaction may also explain the findings of Chernyak and Sobel (2016). They designed a game in which 4-year-old children watched a puppet destroy a tower made by the child and another puppet, either intentionally or accidentally according to experimental condition, while the experimenter was not watching. Then, the experimenter punished the first puppet by not giving it stickers as a prize and instead giving all the stickers to the child. The authors found that children spontaneously shared more stickers with the puppet that accidentally destroyed the tower than with the puppet that intentionally destroyed it, correcting the inequality and unfair punishment given by the experimenter. This was the case even though the same children, when asked to reflect explicitly on the goodness or badness of the people they had helped, tended to judge them according to a typical outcome/intention shift (that is, the youngest children used only the 
outcome in their explicit judgements, while older children used both outcome and intention). Still more direct evidence about the flexible role of intentions in moral judgements comes from a study showing that when asked to judge a character's action, younger children used information about outcomes in judging accidents while older children used information about intentions; but when the question was rephrased to ask instead about the moral worth of the character themselves, even younger children could use information about intentions (Nobes, Panagiotaki \& Bartholomew, 2016). What this change in the phrasing of questions shows is that children can automatically focus on intentions - even positive intentions - in making moral judgements. The outcome-to-intent shift may thus be sensitive to experimental framing, due to the way in which the particular wording of questions directs children's attention either to the agents' moral worth or to the valence of specific actions.

This result was partially replicated in our own recent study (Moreno-Romero \& Ingram, 2019). Children from 3 to 9 years old were asked to provide acceptability and punishment judgments for four different situations that combined intentions and outcomes factorially (i.e., the four combinations of positivity and negativity for both intentions and outcomes); additionally they had to decide how many points they would like to share with the main character in the situation given its behavior, something that can be considered a thirdparty punishment task in which a behavioral reward could be decided on the basis of the moral judgement. The study found that children showed no evidence of an outcome-to-intent shift when asked to make abstract judgements of story characters: from an early age children considered both outcome and intentions, and characters responsible for accidents (with positive intentions but negative outcomes) were judged as better and less deserving of punishment than characters whose negative intentions failed. However, there was some evidence of an outcome-to-intent shift in their behavioral reward decisions: older children exhibited reward decisions in line with their abstract judgements, but younger children's allocations were not congruent with their judgements of goodness and punishment (MorenoRomero \& Ingram, 2019).

Given this evidence from diverse studies, it is clear that inference about both causality and intentionality can be early-developing and presumably often automatic. Therefore, both are probably best modelled as Type 1 processes. As argued above, more recent elaborations of the dual-process approach are based on a tripartite view of the mind, with the automatic part, known as Type 1 processes, divided into a "grab-bag" of innate, maturational responses that are always automatic (mostly designed to direct attention, though also including some basic emotional programs, such as the fear response to a looming physical threat), and a 
portfolio of culturally and experientially acquired skills that are consciously learned but then become automatic through practice, such as writing or riding a bicycle (Evans \& Stanovich, 2013: Stanovich et al., 2011; cf. McCauley’s, 2011, distinction between “maturational” and "practiced" behavior). Type 2 processes, as we have seen, load heavily on executive functioning (in particular, on working memory), override Type 1 processes, and are divided into an algorithmic mind that executes processes of symbolic reasoning, and a reflective mind that decides when to deploy such processes.

Applying this model to the outcome-to-intent shift, it could be that evaluation of negative outcomes involves a Type 1 process that analyzes causality (a simple negative emotional reaction leading to abstract moral judgement and concrete advocation of punishment). Evaluation of failed negative intentions might involve a separate Type 1 process responsible for mindreading, but one that leads to a similarly negative emotional reaction (because of the imagined negative outcome), and again, to an advocation of punishment. The key age difference comes with the evaluation of failed positive intentions (accidental harms). For younger children, depending on the details of the experimental paradigm, either one of these competing Type 1 processes (outcome evaluation or mindreading) may be activated. This is never normally the case for older children and adults, who tend to react more forgivingly to accidents based seemingly on Type 2 processes of mental simulation (in "developed", large-scale societies at least; we return to possible cultural influences on these processes later in the chapter). What then is responsible for this developmental change? In the next section we consider the Type 2, "reflective mind" process of cognitive decoupling, which may be responsible for mediating or arbitrating in some way between the two competing Type 1 processes, eventually leading to a shift in emphasis on intent over outcome.

\section{Cognitive Decoupling and the Articulation of Divergent Intuitions}

While there may be separate Type 1 heuristics that handle outcomes and intentions, what really needs explaining is how during development these heuristics become articulated together, so that in the case of accidents, information about good intentions overrides information about bad outcomes. According to recent dual-process theories, the overriding of intuitive, Type 1 reactions by Type 2 responses can be explained by the operation of a reflective Type 2 process called "cognitive decoupling" (Pennycook, Fugelsang \& Koehler, 2015; Stanovich, 2011; Stanovich \& West, 2008). In simple terms, this is the ability to block 
out emotionally stimulating (i.e., Type 1 process triggering) information and focus on the application of formal rules: "Decoupling occurs when the automatic input-output connections of the brain are suspended while cognitive simulations of hypotheticals are carried out" (Toplak, West, \& Stanovich, 2014, p. 1045). An everyday example would be applying simple algebra to calculate contributions to a restaurant bill when the adult members of an extended family have different numbers of dependents (Kahneman, 2011, reviewed many examples of how the application of formal rules in this type of situation can be impaired by various kinds of biases). The extent to which this process is deployed varies between individuals, which helps to explain individual differences in susceptibility to biases. Moreover, a cognitive developmental study found that the application of cognitive decoupling increased with children's age, as well as being correlated with their intellectual and executive-functioning abilities. (This was measured by performance on a variety of cognitive bias tasks denominator neglect, belief bias, base rate sensitivity, resistance to framing, and "otherside thinking" - where participants had to override a Type 1 response with Type 2 thinking in order to find the correct answer; Toplak et al., 2014.) Therefore, a kind of cognitive decoupling could be responsible for children's tendency to focus more on intentions in moral reasoning as they get older, especially in situations involving an accidental transgression. The idea is that children might learn to block out the information from the Type 1 negative evaluation of outcomes, applying a process of cognitive decoupling to focus on excusing the character's accidental action because of their positive or neutral intent.

Cognitive decoupling can help explain some of the key results from the literature on the outcome-to-intent shift reviewed above. In particular, we want to highlight the findings that (a) the outcome-to-intent shift may not occur at all - or may occur earlier - when children are asked to evaluate agents rather than actions (Moreno-Romero \& Ingram, 2019; Nobes et al., 2016); and that (b) it seems to occur later when analyzed using more behavioral methods, such as asking children how much punishment they think an agent deserves (Cushman et al., 2013), or how many reward points they want to assign them (MorenoRomero \& Ingram, 2019). Both of these findings can be explained using the idea of cognitive decoupling. With (a), when judging actions rather than agents, children need to engage in cognitive decoupling in order to "forgive" an accident, overriding the negative effects that they witness in order to take the positive or neutral intentions into account. In contrast, cognitive decoupling as a Type 2 process may not always "need" to be triggered when judging an agent, since when children are asked questions about an agent they may focus automatically on the agent's intentions and thus their long-term potential for other bad 
behavior, leading to more forgiving judgements about accidents in particular. This may depend on the particular characteristics of the task: for instance, cognitive decoupling could still be needed for designs like that of Piaget, 1932, discussed above, where the direct comparison in the extent of the damage might have made the outcome more salient, explaining why he found an outcome-to-intent shift even though he asked children to judge an agent.

The same sort of idea may apply to finding (b): when making abstract judgements, attention is drawn to abstract concepts that are long-term indicators of character, such as intentions; the valence of the results is not so salient, and thus does not have to be suppressed through cognitive decoupling. On the other hand, when deciding a behavioral response, principles of reciprocity may lead to an impulse to respond "in kind" to the bad outcome of the observed action, and these may have to be overridden by Type 2 processes in order to treat an accident more favorably than an action with a negative intention behind it. What we see, then, is a move from two competing Type 1 processes being triggered by the characteristics of the situation (the way the experiment is set up) to the operation of a Type 2 process that selectively overrides the outcome-responsive process (in the case of accidents) in favor of the intent-responsive process, in favor of algorithmic considerations like normcompliance and consistency.

This analysis is not far from other recent theoretical accounts of the outcome-to-intent shift (reviewed by Margoni \& Surian, 2016), which emphasized that only after developing complex executive functioning (EF) do children treat accidents as less blameworthy in verbal tasks. Such an explanation is needed because of the evidence that from an early age, accidents may be treated more favorably than failed negative attempts in preverbal, "partnerchoice" tasks, where a child had to choose between two toys to play with, each of which were presented as causing different types of accidental or intentional, good or bad outcomes (Van de Vondervoort \& Hamlin, 2018; Woo, Steckler, \& Hamlin, 2017). In common with many accounts of the developmental differences between pre-verbal and verbal false-belief-task performance (reviewed by Apperly, 2012), one tactic has been to say that two- and threeyear-old children "pass" pre-verbal partner-choice tasks but "fail" verbal judgements because of the higher executive functioning demands imposed by the latter type of task.

The problem with invoking EF demand in general as an explanation for such developmental changes, as opposed to cognitive decoupling in particular, is that it is hard for general EF accounts to distinguish between different framings of the experimental tasks involved. For example, it is difficult to use developments in EF to explain the finding that 
children are more tolerant of accidents earlier when asked to judge the agent rather than the action (as seen in the discussion above of the results of Nobes et al., 2016). What is it about asking children to judge an action that would impose more of a demand on EF? Might we not expect judgements of the agent behind the action to be more demanding? However, this problem does not mean that explanations in terms of EF cannot be accommodated to dualprocess accounts. A possible way forward is suggested by the work of Carlson, David and Leach (2005), who divided EF into "hot" and "cold" functions (Metcalfe \& Mischel, 1999), corresponding in our terminology to Type 1 and Type 2 processes. Using a task originally designed for chimpanzees (Boysen \& Berntson, 1995), Carlson and colleagues showed that children were better at solving "Less Is More" problems (which involve asking for a smaller set of rewards in order to get another, larger set) when they had to request abstract symbols (such as Arabic numerals) representing the rewards, than when the request was concretely for the rewards themselves. Symbolic distancing may thus be conceptually related to cognitive decoupling, in that it is necessary to invoke a Type 2 explicit process in order to override the impulse to ask for the bigger reward. It seems moreover that cognitive decoupling may be easier when thinking through symbols, engaging "cold" EF, than when thinking about concrete objects (food rewards, in many cases) and having to engage "hot" EF to override an automatic Type 1 response.

However, what is not often addressed in studies of executive functioning is why we have this kind of Type 2 process that can override Type 1 cognitive biases in the first place, and why it may be triggered more easily in certain, "symbolically distant" tasks than in others (Stanovich, 2009). After all, visual symbols were presumably not present in adaptively relevant environments (Dehaene, Cohen, Morais, \& Kolinsky, 2015). Perhaps, as well as being triggered by symbols, cognitive decoupling may be easier when one is thinking about the intentions and motivations behind people's actions than when one is thinking only about the actions themselves - as in the case of the outcome-to-intent shift. Looked at in evolutionary terms, the application of such thinking to control one's own actions - inhibiting the impulse to punish something which had an unwelcome result, if it did not have a negative intention behind it - could arise from the sort of probabilistic learning process described by Bjorklund (2015). Recall that Bjorklund argued that instinctive responses (Type 1, in our terminology) do not emerge fully-formed in babies, but instead depend on a flexible process of interaction with the environment (in the case of humans, the social and cultural environment). Young children could find that other people react differently if they try to 
punish accidental actions that led to harm, than if they punish actions with negative intentions.

To see how this would work, it is helpful once again to compare reactions to accidents with reactions to failed negative intentions. In our study of the four different possible conditions of positive/negative outcomes/intentions (Moreno-Romero \& Ingram, 2019), perpetrators of accidents were rated less favorably than people who successfully fulfilled positive intentions. In contrast, people who failed to fulfil their negative intentions were evaluated as just as bad as people who successfully fulfilled negative intentions. This could be because of an evolved negativity bias which leads children to be attentive to possible examples of negativity regardless of whether they are witnessed in the form of outcomes or intentions. However, the necessity of finding good cooperative partners might also have selected for an ability to spot positive intentions, explaining the results of Hamlin and colleagues, discussed earlier (Hamlin, 2013; Van de Vondervoort \& Hamlin, 2018) that children are tolerant of accidents in preverbal "partner choice" tasks. Parallel statistical learning processes about the responses to outcomes and intentions would lead to parallel "calibrations" of these Type 1 responses depending on the social environment (reinforcing negative judgements of negative intentions, and positive judgements of positive intentions). Cognitive decoupling might then come into play when children are later asked to make verbal responses. The initial tendency might simply be to give a negative verbal reaction to any negative intention or outcome, due to negativity bias. But processes of calibration might encourage children to modify their Type 1 responses, through Type 2 cognitive decoupling, to make it more consistent with the combination of results for Type 1 processes described for Hamlin and colleagues' preverbal tasks. This process might be easier for agents due to the way that judgements of agents trigger reflective thinking more easily than judgements of actions.

\section{Cultural Variation in the Weight Placed on Intentions}

Viewing cognitive decoupling as tied to an evolved probabilistic learning process also helps to explain why this process can have different outcomes in different cultures. Similarly to the way in which there are cultural differences in the seriousness ascribed to conventional violations (so that in some societies or groups, breaking a conventional rule can be regarded as just as bad as inflicting personal harm on someone; Haidt, Koller, \& Dias, 1993), there are cultural differences in the rules that different groups follow to make moral judgments according to outcomes or intentions. One landmark study of ten different societies (eight 
small-scale, two large-scale) presented adult participants with hypothetical scenarios containing different transgressions (e.g., physical harm, damaging the wellbeing of a group, or a food taboo violation), while systematically varying the intentionality of the perpetrator. The results demonstrated a great deal of cross-cultural variation in the extent to which people assigned importance to intentions, with both rural European and urban North American participants seeing them as critically important to the evaluation of accidents, but other cultures giving them less weight (Barrett et al., 2016). One of the two societies that were least inclined to see intentions as important was the Yasawa culture from Fiji, in the Pacific Islander cultural area. This region was already known in the anthropological literature for its "opacity of mind" norms, which in many contexts prohibit verbal speculation about the internal reasons why other people made particular decisions (Robbins \& Rumsey, 2008).

Thus it may be that in WEIRD (Henrich, Heine, \& Norenzayan, 2010), "mindminded" (Hughes, Devine, \& Wang, 2018) cultures, children are reminded to think about and potentially forgive - accidents from an early age, triggering a routine learned response when they are faced with analyzing an accident in an experiment. In cultures with "opacity of mind" norms they do not receive such feedback, since other people's intentions are not often publicly discussed; thus, even adults in these cultures have a tendency to react to accidents with judgements that happen to be similar to children's intuitive, Type 1 judgements. An alternative possibility, suggested by Saxe (2016), is that while Yasawa adults' public evaluations of the experimental scenarios were congruent with their explicit norms about appropriate public discourse, they diverged from their private cognitions, which implicitly processed the characters' intentions (cf. Astuti, 2015). However, another recent cross-cultural study (using a similar methodology to Barrett et al., 2016) showed that even Yasawan adults could be primed to focus on intentions - by asking them to list thoughts that God would or would not like them to think - which led them to be more forgiving of accidents (McNamara,Willard, Norenzayan \& Henrich, 2019). It seems unlikely that mere priming about "thoughts" was enough to induce participants to abandon their norms against taking others' intentions into consideration and reveal their private analysis of the experimental vignettes: more likely, it prompted them to consider an aspect of the situations in the vignettes that they were overlooking before. The Yasawa don't excuse accidents automatically: their habitual action is to continue condemning them because this has rarely been overridden by explicit instruction. However, they can still be induced to react using a Type 2 process by an experimental prime. This fits with the theoretical conception of cognitive decoupling as a resource that is available to everyone in every situation, whenever 
we want to look at a problem from a different angle. The circumstances that trigger decoupling may include standard routines ingrained within a culture, situational primes, or even original reflections by individuals.

An outline of our model for cultures without opacity of mind norms is summarized in Figure 2 below, which attempts to conceptualize the Type 1/2 typology in an integrated way, rather than simply referring to two different types of processes. Here they are modeled in terms of two different routes of learning, a Type 1 route that uses Bjorklund's (2015) idea of innate attentional biases associated with evolved probabilistic learning mechanisms, and a Type 2 route that uses Stanovich's (2011) distinction between the reflective mind and the algorithmic mind (compare Figure 1 above).

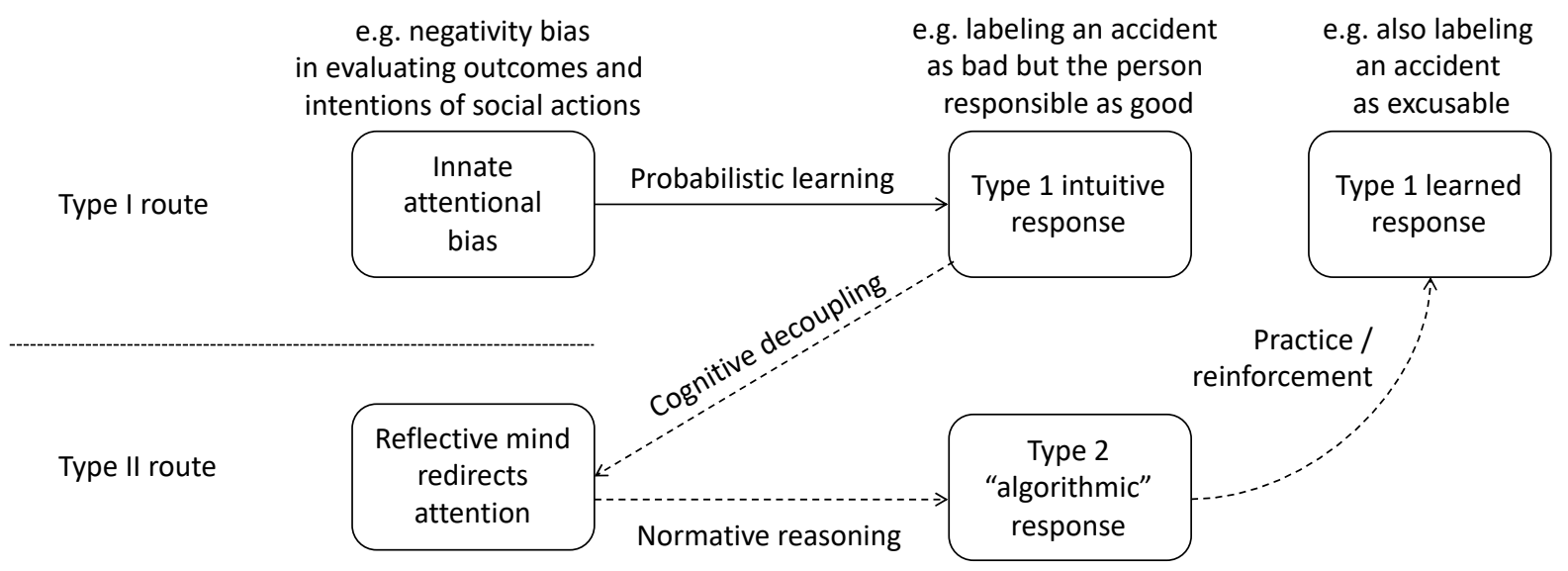

Figure 2. Model of two routes of learning about moral actions.

Children begin social learning about moral actions with a set of innate attentional biases, which cause them to focus on social actions in the first place, but also to pay more attention to negatively valenced actions than to positively valenced actions. Through probabilistic learning they naturally pick up on people's intentions, learning to associate them with outcomes of the equivalent valence. Because the resulting judgements are dependent on attentional focus, they are sensitive to experimental framing: when asked if an action is wrong at an early age, they may focus on the outcome, whereas when asked if an agent is bad, some (but not all) children may focus on the intention earlier on (this may also be the case in partner-choice paradigms). The outcome-to-intent shift thus appears specifically when asked about actions or for behavioral measures, as children grow older and start to use the Type 2 learning route, at least in WEIRD cultural contexts. This involves engaging in cognitive decoupling (perhaps encouraged by the cognitive dissonance between their 
intention-based processing and their outcome-based processing) to reflect on an agent's motives even though they have been asked whether the action was right or wrong. They then apply normative reasoning to come up with a response based on culturally learned moral reasoning algorithms (for example, excusing an accident if a person was not negligent, but not excusing it if they were perceived as negligent; Nobes, Panagiotaki, \& Engelhardt, 2017). Finally, this analysis of and response to accidents can become automaticized as a Type 1 learned response through practice and reinforcement over time, perhaps in an analogous process to the probabilistic learning responsible for the automaticization of Type 1 responses.

However, in some non-WEIRD cultures such as the Yasawa of Fiji, this Type 2 route may be much less common and will not usually become automatic, either because children are not prompted to think so much about intentions, or because they do not have the normative reasoning about accidents readily available to come up with an algorithmic response. The former interpretation would seem to be supported by the fact that excusing accidents could be encouraged by priming about "thoughts" in McNamara and colleagues' (2019) experiment. Thus, cultural differences in weight given to outcome versus intent likely do not represent differences in capacities so much as differences in experience with particular practices and reasoning styles.

\section{Discussion}

We believe that this model is an advance on previous theoretical conceptions of the outcome-to-intent shift. For example, Cushman and colleagues' (2013) account is underspecified in two main ways. First, they introduced the idea of a "bad act detector" to explain why children are sensitive to negative intentions from an early age, without explaining why this would be triggered by negative intentions as well as negative outcomes, when there was no analogous detector for "good acts" that was activated by positive intentions. That does not fit with the evidence from Hamlin's (2013) study that 8-month-old children are capable of taking good intentions into account, in partner choice paradigms. This pattern of results is easily integrated into our model by assuming that being asked to choose a partner may prime children to think more of actors' intentions than the rightness or wrongness of the act itself. Similarly, the notion that only "bad acts" are automatically linked to intentions does not square with the evidence of Nobes et al. (2016) that children evaluate intentions earlier when 
asked about agents than when asked about actions.2 The latter study, using explicit judgements rather than partner choice as an outcome, points to the second area in which Cushman and colleagues' model is underspecified. They explained the change associated with the outcome-to-intent shift as resulting from a "conceptual reorganization within the system of moral reasoning itself; the emergence of an intent-based process for judging moral wrongs" (2013, p. 14), yet did not explain why this would apply earlier to judgements of negative compared to positive intentions, and to judgements of agents compared to actions. In our model, in contrast, this change is conceptualized more as a capability for cognitive decoupling that allows children to step back from focusing on the outcome when evaluating the morality of an action. This explains both why they are capable of preferring accidental to failed intentional violators at younger ages (which again does not fit with the notion of an "internal" moral reorganization at a later age); and why, in certain cultures such as the Yasawa that do not draw children's attention to mental states, they continue judging accidents harshly into adulthood.

A different theoretical account of the outcome-to-intent shift has focused on developments in executive functioning (in common with many explanations of the development of false-belief understanding). According to Margoni and Surian (2016, p. 3): "The additional processing demands of the elicited-response [explicit judgement] tasks compared to the spontaneous-response [partner choice] task used in the infant literature, lead kindergartners to produce outcome-based evaluations." However, this begs the question since they do not explain why outcome-based evaluations would be less demanding on three-yearolds' executive functioning than intent-based evaluations, seeing as eight-month-old infants are capable of both. And, as with that of Cushman et al. (2013), this explanation does not tally with the more recent findings either from Nobes et al. (2016) that accidents are excused much earlier when judging agents than when judging actions, or from Barrett et al. (2018) and McNamara et al. (2019) that adults often fail to take intentions into account in some nonWEIRD societies. Conceptualizing a "failure" to show an outcome-to-intent shift in those societies as the result of a "deficiency" in executive functioning would be unpalatable (see Kidd, Palmeri, \& Aslin, 2013, for a related critique of the universality of the "marshmallow task" as a measure of executive functioning across different cultural environments). Instead, reconceptualizing the broad category of executive functioning in terms of "cognitive

2 In fairness, of course Cushman et al. (2013) would not necessarily have been able to read about these two studies before writing up their own. 
decoupling" or "symbolic distancing" - clearly related to executive functioning, but much more focused in their reference - allows us to understand how different cultural and normative environments (not to mention different experimental framings) might encourage people to a greater or lesser extent to "take a step back" from particular problems and consider them in a detached, abstract way.

As a high-level model of social cognitive development, the dual-process account elaborated in this chapter is not particularly easy to test. Nevertheless, we believe that there are two main ways in which it could be examined empirically. Firstly, since excusing accidents is not universal, even among WEIRD children, micro-genetic longitudinal studies of development could test whether there is developmental continuity between participants who tend to excuse accidents as infants (when framed in terms of partner choice) and those who excuse them as older children performing explicit judgement tasks, after the outcome-tointent shift has taken place. "Continuity" accounts that stress executive functioning (e.g., Margoni \& Surian, 2016) would predict that the same children should be more likely to excuse accidents in both paradigms. In contrast, our dual-process account would predict that these children might be different individuals, since infants excuse accidents using the Type 1 route (meaning that those infants more susceptible to framing effects should be more likely to excuse them), and older children excuse them using the Type 2 route (meaning that those children who engage in more cognitive decoupling should be more likely to excuse them). This could be tested along with appropriate covariates (framing sensitivity and executivefunctioning measures of cognitive decoupling, respectively). The predictive value of the model could also be tested on adults by putting them under high cognitive load while judging accidents and failed intentional violations. Our model would predict that when under cognitive load even WEIRD adults should often revert to more childlike, outcome-based Type 1 judgements, at least in novel situations (unless they have a lot of experience with automatically judging accidents), since cognitive decoupling happens less frequently under stress. This is not a prediction of models which explain the outcome-to-intent shift in terms either of a conceptual reorganization in the moral domain, or the development of new executive-functioning skills.

\section{Conclusion}

One of our main aims in this chapter has been to show with a developmental example how Type 1 and Type 2 processes are not always opposed to each other but can interact in interesting ways in development. Recent empirical work shows that the textbook idea of the 
outcome-to-intent shift as a developmental change from exclusively using outcomes in moral judgements, to exclusively using intentions, is incorrect. Children take both outcomes and intentions into account from an early age, and gradually learn to integrate them in their verbal judgements and controlled behavioral responses. What has rarely been explained in the literature is the contradiction between the classic Piagetian studies showing an absence of consideration of intentions in young children under certain experimental paradigms, and the newer results showing that they do take intentions into account under other paradigms. Our contribution here has been to map out a way of reconciling those divergent sets of results, with a dual-process model in which the Type 2 process of cognitive decoupling overrides one Type 1 response in favor of another. The way in which this happens may be analogous to Railton's (2014) idea of attunement. Just as his lawyer was unsatisfied with the jury's response to her habitual, formal style of argumentation, and engaged her reflective mind to override that in favor of a more intuitive approach, so children who are faced with normative pressure to forgive accidents may override an intuitive outcome-based response in favor of an equally intuitive, but situationally weaker, intent-based response. Comparing those two examples also underlines that Type 1 processes are not always innate: in fact, as Bjorklund's (2015) idea of evolved probabilistic learning mechanisms suggests, instincts are always somewhat flexible and sensitive to experience.

Applying this idea to evolutionary ethics is valuable because it can help us get away from the assumption that automatic, Type 1 moral reactions are "irrational" relics of an evolved past, and necessarily inferior to more reflective, Type 2 moral responses (see Pennycook et al., 2018). In reality, moral dilemmas such as weighing outcome against intent probably set off conflicting Type 1 intuitions. There is individual, situational and cultural variation in which of these intuitions wins out, and some of that variation may be caused by differences in the tendency to use Type 2 processes to inhibit an automatic response and engage in formal, algorithmic reasoning. Nevertheless, these Type 2 processes also have an evolutionary rationale, in terms of helping us find a socially acceptable response in the context of the normative environment in which we find ourselves.

\section{References}

Apperly, I. A. (2012). What is "theory of mind"? Concepts, cognitive processes and individual differences. Quarterly Journal of Experimental Psychology, 65, 825-839.

Astuti, R. (2015). Implicit and explicit theory of mind. Anthropology of this Century, 13, 636650.

Barrett, H. C., Bolyanatz, A., Crittenden, A. N., Fessler, D. M., Fitzpatrick, S., Gurven, M., et al. (2016). Small-scale societies exhibit fundamental variation in the role of intentions 
in moral judgment. Proceedings of the National Academy of Sciences, 113, 46884693.

Barrouillet, P. (2011). Dual-process theories of reasoning: The test of development. Developmental Review, 31, 151-179.

Baylor, A. L. (2001). A U-shaped model for the development of intuition by level of expertise. New Ideas in Psychology, 19, 237-244.

Bélanger, N. D., \& Desrochers, S. (2001). Can 6-month-old infants process causality in different types of causal events? British Journal of Developmental Psychology, 19, $11-21$.

Berg-Cross, L. G. (1975). Intentionality, degree of damage, and moral judgments. Child Development, 46, 970-974.

Bjorklund, D. F. (2015). Developing adaptations. Developmental Review, 38, 13-35.

Bjorklund, D. F., \& Pellegrini, A. D. (2000). Child development and evolutionary psychology. Child Development, 71, 1687-1708.

Bjorklund, D. F., \& Pellegrini, A. D. (2002). The origins of human nature: Evolutionary developmental psychology. American Psychological Association.

Boysen, S. T., \& Berntson, G. G. (1995). Responses to quantity: Perceptual versus cognitive mechanisms in chimpanzees (Pan troglodytes). Journal of Experimental Psychology: Animal Behavior Processes, 21, 82-86.

Brakefield, P. M. (2006). Evo-devo and constraints on selection. Trends in Ecology and Evolution, 21, 362-368.

Carroll, S. B. (2005). Endless forms most beautiful: The new science of evo devo and the making of the animal kingdom. Norton.

Carlson, S. M., Davis, A. C., \& Leach, J. G. (2005). Less is more: Executive function and symbolic representation in preschool children. Psychological Science, 16, 609-616.

Chernyak, N., \& Sobel, D. M. (2016). "But he didn't mean to do it": Preschoolers correct punishments imposed on accidental transgressors. Cognitive Development, 39, 13-20.

Cicchino, J. B., \& Rakison, D. H. (2008). Producing and processing self-propelled motion in infancy. Developmental Psychology, 44, 1232-1241.

Cohen, L., \& Amsel, G. (1998). Precursors to infants' perception of the causality of a simple event. Infant Behavior and Development, 21, 713-732.

Constantin, S. R. (2014). Do rational people exist? https://srconstantin.wordpress.com/2014/06/09/do-rationalists-exist/

Cushman, F. (2013). Action, outcome, and value: A dual-system framework for morality. Personality and Social Psychology Review, 17, 273-292.

Cushman, F., Sheketoff, R., Wharton, S., \& Carey, S. (2013). The development of intentbased moral judgment. Cognition, 127, 6-21.

Dehaene, S., Cohen, L., Morais, J., \& Kolinsky, R. (2015). Illiterate to literate: Behavioural and cerebral changes induced by reading acquisition. Nature Reviews Neuroscience, 16, 234-244.

Evans, J. S. B. (2011). Dual-process theories of reasoning: Contemporary issues and developmental applications. Developmental Review, 31, 86-102.

Evans, J. S. B. (2012) Dual process theories of deductive reasoning: Facts and fallacies. In K. J. Holyoak \& R. G. Morrison (Eds.), The Oxford handbook of thinking and reasoning (pp. 115-133). Oxford University Press.

Evans, J. S. B. (2020). Hypothetical thinking: Dual processes in reasoning and judgement. Psychology Press (Classic Ed.).

Evans, J. S. B., \& Stanovich, K. E. (2013). Dual-process theories of higher cognition: Advancing the debate. Perspectives on Psychological Science, 8, 223-241. 
Farroni, T., Massaccesi, S., Menon, E., \& Johnson, M. H. (2007). Direct gaze modulates face recognition in young infants. Cognition, 102, 396-404.

Graham, J., Haidt, J., Koleva, S., Motyl, M., Iyer, R., Wojcik, S. P., \& Ditto, P. H. (2013). Moral foundations theory: The pragmatic validity of moral pluralism. Advances in Experimental Social Psychology, 47, 55-130.

Grosse Wiesmann, C., Friederici, A. D., Singer, T., \& Steinbeis, N. (2017). Implicit and explicit false belief development in preschool children. Developmental Science, 20, e12445.

Haidt, J., Koller, S. H., \& Dias, M. G. (1993). Affect, culture, and morality, or is it wrong to eat your dog? Journal of Personality and Social Psychology, 65, 613-628.

Hamlin, J. K. (2013). Failed attempts to help and harm: Intention versus outcome in preverbal infants' social evaluations. Cognition, 128, 451-474.

Hebble, P. W. (1971). The development of elementary school children's judgment of intent. Child Development, 42, 1203-1215.

Helwig, C. C., Zelazo, P. D., \& Wilson, M. (2001). Children's judgments of psychological harm in normal and noncanonical situations. Child Development, 72, 66-81.

Henrich, J., Heine, S. J., \& Norenzayan, A. (2010). Most people are not WEIRD. Nature, 466, 29.

Hughes, C., Devine, R. T., \& Wang, Z. (2018). Does parental mind-mindedness account for cross-cultural differences in preschoolers' theory of mind?. Child development, 89(4), 1296-1310.

Irons, W. (1998). Adaptively relevant environments versus the environment of evolutionary adaptedness. Evolutionary Anthropology, 6, 194-204.

Kahneman, D. (2011). Thinking, fast and slow. New York: Farrar, Strauss, \& Giroux.

Karmiloff-Smith, A. (1992). Beyond modularity: A developmental perspective on cognitive science. Cambridge, MA: Bradford.

Keren, G., \& Schul, Y. (2009). Two is not always better than one: A critical evaluation of two-system theories. Perspectives on Psychological Science, 4, 533-550.

Kidd, C., Palmeri, H., \& Aslin, R. N. (2013). Rational snacking: Young children's decisionmaking on the marshmallow task is moderated by beliefs about environmental reliability. Cognition, 126, 109-114.

King, M. (1971). The development of some intention concepts in young children. Child Development, 1145-1152.

Kulke, L., Reiß, M., Krist, H., \& Rakoczy, H. (2018). How robust are anticipatory looking measures of Theory of Mind? Replication attempts across the life span. Cognitive Development, 46, 97-111.

Kushnir, T., Xu, F., \& Wellman, H. (2010). Young children use statistical sampling to infer the preferences of other people. Psychological Science, 21, 1134-1140.

Lickliter, R., \& Honeycutt, H. (2013). A developmental evolutionary framework for psychology. Review of General Psychology, 17, 184-189.

LoBue, V., \& Rakison, D. H. (2013). What we fear most: A developmental advantage for threat-relevant stimuli. Developmental Review, 33, 285-303.

Margoni, F., \& Surian, L. (2016). Explaining the U-shaped development of intent-based moral judgments. Frontiers in Psychology, 7, 1-6.

McCauley, R. N. (2011). Why religion is natural and science is not. Oxford University Press.

McNamara, R. A., Willard, A. K., Norenzayan, A., \& Henrich, J. (2019). Weighing outcome vs intent across societies: How cultural models of mind shape moral reasoning. Cognition, 182, 95-108.

Melnikoff, D. E., \& Bargh, J. A. (2018). The mythical number two. Trends in Cognitive Sciences, 22, 280-293. 
Metcalfe, J., \& Mischel, W. (1999). A hot/cool-system analysis of delay of gratification: dynamics of willpower. Psychological Review, 106, 3-19.

Mikhail, J. (2007). Universal moral grammar: Theory, evidence and the future. Trends in Cognitive Sciences, 11, 143-152.

Moreno-Romero, C. O., \& Ingram, G. P. D. (2019). Children consider both outcome and intent in moral judgements, punishment recommendations, and reward allocations. Unpublished manuscript. Preprint available at https://psyarxiv.com/zpv6h

Muentener, P., \& Bonawitz, E. B. (2017). The development of causal reasoning. In M. R. Waldmann (Ed.), The Oxford handbook of causal reasoning (pp. 677-698). Oxford University Press.

Nobes, G., Panagiotaki, G., \& Bartholomew, K. J. (2016). The influence of intention, outcome and question-wording on children's and adults' moral judgments. Cognition, 157, 190-204.

Nobes, G., Panagiotaki, G., \& Engelhardt, P. E. (2017). The development of intention-based morality: The influence of intention salience and recency, negligence, and outcome on children's and adults' judgments. Developmental Psychology, 53, 1895-1911.

Panksepp, J. (2005). Affective consciousness: Core emotional feelings in animals and humans. Consciousness and Cognition, 14, 30-80.

Pauls, F., Macha, T., \& Petermann, F. (2013). U-shaped development: An old but unsolved problem. Frontiers in Psychology, 4, 301.

Pennycook, G., De Neys, W., Evans, J. S. B., Stanovich, K. E., \& Thompson, V. A. (2018). The mythical dual-process typology. Trends in Cognitive Sciences 22, 8, 667-668.

Pennycook, G., Fugelsang, J. A., \& Koehler, D. J. (2015). What makes us think? A threestage dual-process model of analytic engagement. Cognitive Psychology, 80, 34-72.

Perner, J., \& Roessler, J. (2012). From infants' to children's appreciation of belief. Trends in Cognitive Sciences, 16, 519-525.

Piaget, J. (1932). The moral judgment of the child. Free Press (M. Gabain, Trans.).

Railton, P. (2014). The affective dog and its rational tale: Intuition and attunement. Ethics, 124, 813-859.

Robbins, J., \& Rumsey, A. (2008). Introduction: Cultural and linguistic anthropology and the opacity of other minds. Anthropological Quarterly, 81, 407-420.

Rochat, P. (2015). Layers of awareness in development. Developmental Review, 38, 122-145.

Saxe, R. (2016). Moral status of accidents. Proceedings of the National Academy of Sciences, 113, 4555-4557.

Schneider, D., Slaughter, V. P., \& Dux, P. E. (2017). Current evidence for automatic Theory of Mind processing in adults. Cognition, 162, 27-31.

Scott, R. M., \& Baillargeon, R. (2017). Early false-belief understanding. Trends in Cognitive Sciences, 21, 237-249.

Shiffrin, R. M., \& Schneider, W. (1977). Controlled and automatic human information processing: II. Perceptual learning, automatic attending and a general theory. Psychological Review, 84, 127-190.

Shultz, T. R., Wright, K., \& Schleifer, M. (1986). Assignment of moral responsibility and punishment. Child Development, 57, 177-184.

Siegler, R. S. (2004). U-shaped interest in U-shaped development and what it means. Journal of Cognition and Development, 5, 1-10.

Stanovich, K. E. (2009). What intelligence tests miss: The psychology of rational thought. Yale University Press.

Stanovich, K. E. (2011). Rationality and the reflective mind. Oxford University Press.

Stanovich, K. E., \& West, R. F. (2008). On the relative independence of thinking biases and cognitive ability. Journal of Personality and Social Psychology, 94, 672-695. 
Stanovich, K. E., West, R. F., \& Toplak, M. E. (2011). The complexity of developmental predictions from dual process models. Developmental Review, 31, 103-118.

Suhler, C. L., \& Churchland, P. (2011). Can innate, modular "foundations" explain morality? Challenges for Haidt's moral foundations theory. Journal of Cognitive Neuroscience, 23, 2103-2116.

Tomasello, M. (2019). Becoming human: A theory of ontogeny. Belknap Press.

Tooby, J., \& Cosmides, L. (2010). Groups in mind: The coalitional roots of war and morality. In H. Høgh-Olsen (Ed.,), Human morality and sociality: Evolutionary and comparative perspectives (pp. 191-234). Red Globe Press.

Toplak, M. E., West, R. F., \& Stanovich, K. E. (2014). Rational thinking and cognitive sophistication: Development, cognitive abilities, and thinking dispositions. Developmental Psychology, 50, 1037-1048.

Van de Vondervoort, J. W., \& Hamlin, J. K. (2018). Preschoolers focus on others' intentions when forming sociomoral judgments. Frontiers in Psychology, 9, 1851.

Woo, B. M., Steckler, C. M., Le, D. T., \& Hamlin, J. K. (2017). Social evaluation of intentional, truly accidental, and negligently accidental helpers and harmers by 10 month-old infants. Cognition, 168, 154-163.

Woodward, A. L. (2009). Infants' grasp of others' intentions. Current Directions in Psychological Science, 18, 53-57.

Wu, R., Gopnik, A., Richardson, D. C., \& Kirkham, N. Z. (2011). Infants learn about objects from statistics and people. Developmental Psychology, 47, 1220-1229.

Zelazo, P. D., Helwig, C. C., \& Lau, A. (1996). Intention, act, and outcome in behavioral prediction and moral judgment. Child Development, 67, 2478-2492. 